\title{
Diaǵnóstico de la implementación del SG-SST en las constructoras pioneras de Colombia*
}

\author{
Diagnosis of the OHSMS implementation in the pioneering \\ construction companies of Colombia
}

\section{Diagnóstico da implementação do SG-SST nas empresas construtoras pioneiras da Colômbia}

\section{Yessica Idalides Barrios Pajaro*}

Universidad Militar Nueva Granada

Cómo citar este artículo: Barrios-Pájaro, Y. I., (2020). Diagnóstico de la implementación del SG-SST en las constructoras pioneras de Colombia. Signos, Investigación en Sistemas de Gestión, 12(2), 149-159. Dor: https://doi.org/10.15332/24631140.5943

\section{RESUMEN}

En la búsqueda de una metodología gerencial ideal que permita especialmente a las constructoras de Colombia apropiarse de una herramienta eficaz que les garantice una adecuada implementación del Sistema de Gestión de la Seguridad y Salud en el Trabajo SG-SST en los proyectos que desarrollan, se establece el desarrollo de un diagnóstico basado en cuatro enfoques como primer objetivo de la investigación denominada "Metodología gerencial para la implementación del SG-SST en las firmas constructoras pioneras de Colombia". La muestra de la investigación comprende las constructoras pioneras de Colombia, bajo una entrevista con los lineamientos de

\footnotetext{
Artículo de resultado de reflexión.

** Candidata a Magíster en Gerencia de Proyectos. Administradora de Empresas Dual, Gestión y Proyectos, Universidad Militar Nueva Granada. Bogotá D.C., Colombia. Correo electrónico: yesiebarrios@gmail.com y u21100126@unimilitar.edu.co. Este artículo es un resultado parcial de la investigación denominada "Metodología gerencial para la implementación del SG-SST en las firmas constructoras pioneras de Colombia".
} 
la NTC-ISO 45001:2018 y la gerencia de proyectos, con el fin de extraer información que permita la consolidación de una metodología efectiva para la implementación del SG-SST.

Palabras clave: constructoras, gerencia de proyectos, sistema de gestión de seguridad y salud en el trabajo (SG-SST), NTC-ISO 45001:2018.

\section{ABSTRACT}

In the search for an ideal management methodology that allows especially the construction companies in Colombia to appropriate an effective tool that ensure an adequate Occupational Safety and Health Management System implementation in the projects they develop, the first objective of the research called "Management methodology for the implementation of the OHSMS in the pioneering construction companies in Colombia", is the development of a diagnosis based on four approaches. The sample of the research are the pioneer construction companies of Colombia, which is conducted under an interview following the guidelines of the NTC-ISO 45001:2018 and the project management, with the purpose of gathering information that allows the consolidation of an effective methodology for the OSH implementation.

Keywords: OSH Management System, NTC-ISO 45001:2018, project management, construction companies.

\section{RESUMO}

$\mathrm{Na}$ procura de uma metodologia de gestão ideal que permita, especialmente às empresas de construção na Colômbia, apropriarem-se de uma ferramenta eficaz que garanta uma implementação adequada do Sistema de
Gestão de Segurança e Saúde no Trabalho nos projetos por elas desenvolvidos, o primeiro objetivo da pesquisa denominada "Metodologia de gestão para a implementação do SGSST nas empresas de construção pioneiras na Colômbia", é o desenvolvimento de um diagnóstico baseado em quatro aproximações. Como amostra da pesquisa, ela é executada nas empresas de construção pioneiras da Colômbia, de acordo com a revisão feita das diretrizes do NTC-ISO 45001:2018 e a gestão do projeto, a fim de extrair informações que permitam a consolidação de uma metodologia eficaz para a implementação do SG-SST.

Palavras-chave: SG-SST (Sistema de gestão de segurança e saúde no trabalho), NTC-ISO 45001:2018, gestão de projetos, empresas construtoras.

\section{INTRODUCCIÓN}

La gestión de seguridad y salud en el trabajo (SST) se puede definir como un sistema que busca prevenir los incidentes 0 accidentes laborales, disminuir las enfermedades producidas por el desarrollo de una labor y establecer un ambiente general seguro y agradable para el empleado. Por otra parte, el sector de la construcción es, sin duda, protagonista en el desarrollo de las sociedades, ya que es responsable directo de la creación de infraestructura de vivienda, transporte, instalaciones sanitarias, entre otros proyectos, en las que se gesta la cultura y el crecimiento económico de la humanidad (Acevedo, Vásques, \& Ramírez, 2012)

Han sido muchos los estudios que se han desarrollado y muchas las prácticas que se han implementado para mitigar los accidentes de trabajo en el área de la construcción; sin embargo, las evidencias muestran que la gestión en seguridad y salud en el trabajo es muy deficiente (Alarcón, Acuña, Diethelm y Eugenio Pellicer, 2016). Por esta razón, una revisión sistemática de los estudios 
enfocados en la seguridad y la salud en el trabajo es de suma importancia para facilitar y analizar el intercambio de resultados de las investigaciones y el acceso a las tendencias futuras de investigación de la seguridad en la construcción (Zhou, Goh, \& Li, 2014).

En Colombia, el sector de la construcción se mantuvo como uno de los principales motores de crecimiento económico para el país hasta principios del 2015 (Vicepresidencia Gobierno de Colombia, 2015); no obstante, desde finales del mismo año y hasta el primer semestre del 2018, el desempeño del sector presentaba contracciones a ritmos de $-8,6 \%$ anual (vs. $-0,4 \%$ un año atrás), siendo uno de los sectores con peor desempeño a nivel nacional (Clavijo, 2018).

El aumento de la tasa de accidentalidad —6,02\%, para 2009, vs. 6,47\%, para 2017 (SafetYA, 2018)—, el derrumbe de estructuras por errores en los procesos constructivos —edificio Space en Medellín, 2013; edificio en Cartagena, 2017; puente peatonal en Bogotá, 2015; estadio de futbol en Neiva, 2016; edificio en Buenaventura, 2017; puente de Chirajara, 2018 ("Top 5: derrumbes...", 2018) - y el registro de 9.690 enfermedades calificadas como de origen laboral ("Así quedó el balance de accidentes...", 2018) son algunas de las variables que indican que algo está fallando en el desarrollo de los sistemas de gestión de salud y seguridad en el trabajo (SG-SST) de Colombia, reflejándose además como las causas y los efectos de un problema que encierra la falta de incursionar en metodologías.

Con base en esta hipótesis, y en relación con una breve revisión literaria acerca del SG-SST, se destacan tres posiciones que avalan y apoyan el desarrollo de la investigación:

1. La investigación realizada por Cagno, Micheli, Masi y Jacinto (2012) muestra una evaluación económica de los SG-SST en la última década, en la que concluyen que sin importar la complejidad o la sencillez del sistema o de la compañía, el enfoque de la seguridad y salud en el trabajo necesita más investigación multidisciplinaria.

2. Como segunda posición, se encuentra una revisión de la literatura en la evaluación de la madurez de la gestión de sST, realizada sobre 38 artículos publicados entre 2001 y 2017. En este, Rodríguez, Pedraza y Martínez (2017) concluyen lo siguiente: "Aunque se lograron definir diez dimensiones para evaluar la madurez de esta gestión, se requiere dar continuidad a la investigación con el desarrollo de instrumentos y escalas confiables que permitan medir la madurez de esta gestión en las organizaciones" (p. 123).

3. Por último, se destaca la investigación de Erazo y Rodríguez-Rojas (2016), en la que afirman lo siguiente: "existen intervalos vacíos a nivel teórico y conceptual, en cuanto a los procesos que no solo impactan el hacer, sino también la evaluación de indicadores" (p. 67). Es así como, a través de una reunión de expertos, los autores logran construir un instrumento de acuerdo con el draft de la norma técnica NTC-ISO 45001:2018, para la medición de la gestión de SST.

Tomando como base estas posiciones, se considera de gran importancia la elaboración de un diagnóstico acerca de la implementación del SG-SST en las constructoras con mayor antigüedad, con gran importancia y reconocimiento en Colombia, ya que a partir de este diagnóstico se podrá planear y sugerir una metodología efectiva para la implementación del SG-SST en cada proyecto que estas compañías ejecutan.

En este artículo se evidenciará el cumplimiento del primer objetivo de la investigación, en el que, a través de las preguntas planteadas de la entrevista realizada a tres de las cinco constructoras pioneras de Colombia, se logrará percibir y concluir el grado de madurez y de implementación del SG-SST y las metodologías usadas, junto con un importante listado de lecciones aprendidas y mejores prácticas que, con los años de experiencia, han compilado como memoria histórica de cada organización (“Top 5...”, 2020). 


\section{METODOLOGÍA}

El diagnóstico del SG-SST de las constructoras colombiana objeto de este estudio se realizó a través de una entrevista estructurada, validada por tres expertos antes de su aplicación. En la tabla 1, se muestra la estructuración de dicha encuesta.

Tabla 1. Estructura de la entrevista

\begin{tabular}{|c|c|c|}
\hline N. de pregunta & Enfoque & Contenido \\
\hline 1 & SG-SST & ¿Cómo inició la implementación del SG-SST dentro de la organización? \\
\hline 2 & SG-SST & $\begin{array}{l}\text { ¿Bajo qué norma, ley, política o enfoque está fundamentada la implementación del SG-SST } \\
\text { dentro de la organización? }\end{array}$ \\
\hline 3 & Gerencia de proyectos & $\begin{array}{l}\text { ¿Considera usted que la implementación del SG-SST en los proyectos que desarrolla la } \\
\text { organización es exitosa? ¿Por qué? En caso de ser afirmativa la respuesta, ¿cuáles cree usted } \\
\text { que son los factores de éxito? }\end{array}$ \\
\hline 4 & SG-SST & $\begin{array}{l}\text { ¿Considera usted que la implementación del SG-SST ha permitido mejorar en la compañía sus } \\
\text { indicadores, y esta mejora ha permitido fortalecer la toma de decisiones? }\end{array}$ \\
\hline 5 & SG-SST & ¿Cuál es el alcance y los objetivos del SG-SST en los proyectos que desarrolla la constructora? \\
\hline 6 & Organizacional & $\begin{array}{l}\text { Además de la seguridad y la salud, ¿la organización integra otros aspectos que beneficien el } \\
\text { bienestar de los trabajadores? }\end{array}$ \\
\hline 7 & Gerencia de proyectos & $\begin{array}{l}\text { ¿Cuáles son los factores internos y externos que fueron considerados para desarrollar e } \\
\text { implementar el SG-SST? ¿Cómo son seleccionados dichos factores de acuerdo con el desarrollo } \\
\text { de cada proyecto que la constructora desarrolla? }\end{array}$ \\
\hline 8 & Organizacional & $\begin{array}{l}\text { ¿Cómo son designados los roles y responsabilidades dentro del SG-SST en la organización y en } \\
\text { los proyectos que desarrolla? }\end{array}$ \\
\hline 9 & SG-SST & $\begin{array}{l}\text { De acuerdo con las experiencias vividas, ¿considera usted importante que la compañía } \\
\text { cuente con este SG-SST? ¿Por qué? }\end{array}$ \\
\hline 10 & SG-SST & Si no contara con un SG-SST, ¿cuáles cree que serían las consecuencias? \\
\hline 11 & Gerencia de proyectos & $\begin{array}{l}\text { ¿De qué forma es comunicado y divulgado el SG-SST dentro de los proyectos que la } \\
\text { constructora implementa? }\end{array}$ \\
\hline 12 & Metodologías gerenciales & $\begin{array}{l}\text { ¿El responsable de gerenciar los proyectos dentro de la compañía involucra o aplica algún } \\
\text { tipo de metodología para implementar el SG-SST de acuerdo con el tipo de proyecto que la } \\
\text { constructora implementa? }\end{array}$ \\
\hline
\end{tabular}

Fuente: elaboración propia.

La aplicación de la entrevista fue realizada durante los meses de junio, julio y agosto del 2019, tomando en cuenta la disponibilidad de los directores, coordinadores y supervisores de las áreas de SST de cada constructora. De las cinco constructoras pioneras establecidas como muestra de la investigación, solo tres acogieron la convocatoria, consideradas suficientes de acuerdo con el planteamiento de Hernández-Sampieri y Mendoza (2018): "en los estudios cualitativos el tamaño de la muestra no es importante desde una perspectiva probabilística, pues el interés del investigador no es generalizar los resultados de un estudio a una población más amplia, sino profundizar en el entendimiento de un fenómeno" (p. 424). 
La identidad de las constructoras que sí atendieron a la aplicación del instrumento se mantendrá confidencial durante el desarrollo de los resultados, por solicitud de las mismas compañías, por lo que serán identificadas como C1, C2 y C3.

\section{RESULTADOS Y DISCUSIÓN}

Antes de iniciar el análisis de las respuestas dadas por los expertos entrevistados, es necesario que se tenga un referente del tipo de constructoras que se valora para el desarrollo de la investigación; en la tabla 2, se evidencian algunas características:

Tabla 2. Características de las constructoras pioneras entrevistadas

\begin{tabular}{|l|l|l|l|}
\hline Característica & \multicolumn{1}{|c|}{ C1 } & \multicolumn{1}{c|}{ C2 } \\
\hline Años de experiencia & 26 años & 40 años & Más de 60 años \\
\hline Portafolio de servicios & $\begin{array}{l}\text { Vivienda, proyectos de comercio, } \\
\text { edificaciones para oficinas, } \\
\text { educación }\end{array}$ & $\begin{array}{l}\text { Vivienda, construcciones a } \\
\text { terceros, infraestructura }\end{array}$ & Vivienda, locales comerciales \\
\hline Presencia a nivel internacional & Sí & Sí & No registra \\
\hline Cantidad de viviendas entregadas & 7.774 & 45.000 & Más de 2.000 \\
\hline Empleos directos & 1.764 & 1.000 & 800 \\
\hline $\begin{array}{l}\text { Datos de la persona que atiende la } \\
\text { entrevista }\end{array}$ & $\begin{array}{l}\text { Director de seguridad y salud en } \\
\text { el trabajo y medio ambiente; } 5 \\
\text { años en la compañía }\end{array}$ & $\begin{array}{l}\text { Coordinador HSE; 14 años en la } \\
\text { compañía }\end{array}$ & $\begin{array}{l}\text { Coordinador sST; } 9 \text { años en la } \\
\text { compañía }\end{array}$ \\
\hline
\end{tabular}

Fuente: elaboración propia.

Tras el diagnóstico realizado, se compilan las respuestas de los aportes y participaciones más representativas por cada pregunta, que serán de gran importancia para la consolidación de la metodología y la propuesta que reposará como una herramienta importante para todas las organizaciones que desarrollen y ejecuten proyectos con SG-SST.

\section{Enfoque del Sistema de gestión de seguridad y salud en el trabajo}

Las preguntas enfocadas al SG-SST permiten obtener los siguientes resultados, enmarcados como opiniones, observaciones y apuntes de gran interés que las constructoras han tomado como lecciones aprendidas durante toda su experiencia en el sector: a. El inicio de la implementación del SG-SST surge a partir de las exigencias legales o normativas:

C1: "Iniciamos haciendo un autodiagnóstico, como lo decía la resolución en su momento.

C2: "Desde el 2005, la compañía empieza a trabajar el tema pionero de los sistemas de gestión, a partir de las exigencias legales".

C3: "[Por medio de] procesos nuevos o reformas [...] hoy en día, la misma normativa colombiana nos ha llevado a manejar todo como un sistema de gestión".

b. Las tres constructoras destacan como legislación fundamental para el desarrollo de la implementación del SG-SST, el Decreto 1072 de 2015, particularmente el capitulo 6 "que tiene por 
objeto definir las directrices de obligatorio cumplimiento para implementar el Sistema de Gestión de la Seguridad y Salud en el Trabajo (SG-SST)" (Ministerio de Trabajo, 2015). Sin embargo, y a pesar de la presencia internacional de algunas de estas constructoras, estas manifiestan no tener interés por conocer los lineamientos de la nueva NTC-ISO 45001:2018:

C1: "Nosotros nos regimos netamente por el sistema de gestión del Ministerio de Trabajo; la constructora no tiene intención de certificarse bajo ninguna norma internacional como la ISO, bien sea 45001 o 14001 Ambiental".

C3: "Como tal, hemos tomado en cuenta la NTC-ISO 45001:2018. La única que han implementado a nivel de ISO es la 9000, que ya cambió; pero en cuanto a salud y medio ambiente, no".

c. La implementación de un SG-SST permite mejorar la gestión de los procesos en la organización, ya que lleva a que las compañías generen indicadores y establezcan planes de mejoramiento para su crecimiento continuo; en este aspecto, las constructoras manifiestan con certeza la importancia de contar con un SG-SST .
C1: "Sí, totalmente. En el sistema de gestión hay una etapa para planear y otra para verificar y actuar. Si tú no mides estos procesos, no vas a poder saber cuál ha sido el impacto del sistema de gestión".

C2: "Nosotros trabajamos con unos históricos, en temas de accidentalidad, indicadores de desempeño ambientales, calidad integrado al servicio al cliente, que permiten ver cómo a través de los años esos indicadores van mejorando".

C3: "Sí, claro. Todo lo que se desarrolla o todo lo que se implementa va enfocado a eso".

d. El establecimiento de un alcance y objetivos claros para el desarrollo de un SG-SST es de vital importancia; las constructoras aquí valoradas reconocen la necesidad de estar desde el inicio o incluso antes del arranque del proyecto hasta el final o más allá, tal como se muestra en la tabla 3.

e. Cuando se indaga sobre la importancia del SGSST de acuerdo con las experiencias vividas por cada constructora, todas afirmaron que el tenerlo ha influido positivamente en el desarrollo de sus actividades y, sobre todo, en la disminución de accidentalidad, tal como lo expresa la C1: "Sí,

Tabla 3. Alcance y objetivos del SG-SST

\begin{tabular}{|l|l|l|l|}
\hline $\begin{array}{l}\text { Aspecto } \\
\text { valorado }\end{array}$ & \multicolumn{1}{|c|}{ C1 } & \multicolumn{1}{c|}{ C2 } & \multicolumn{1}{c|}{ C3 } \\
\hline Alcance & $\begin{array}{l}\text { Desde la construcción de la sala de ventas } \\
\text { hasta la administración provisional. }\end{array}$ & $\begin{array}{l}\text { Desde la estructuración del proyecto } \\
\text { hasta la entrega final. }\end{array}$ & $\begin{array}{l}\text { El alcance de nuestra área en los } \\
\text { proyectos tiene lugar hasta terminar } \\
\text { proyectos. }\end{array}$ \\
\hline Objetivos & $\begin{array}{l}\text { - Reducir la tasa de accidentalidad. } \\
\text { Lograr un complimiento del } 75 \% \text { de } \\
\text { implementación del programa de } \\
\text { construcción libre de riesgos. } \\
\text { Implementar Focus (Fomentando } \\
\text { cultura de seguridad). } \\
\text { Obtener calificación del } 90 \% \text { de las } \\
\text { auditorías internas para los contratistas. }\end{array}$ & $\begin{array}{l}\text { Garantizar la prevención } \\
\text { de accidentes de trabajo y } \\
\text { enfermedades laborales. } \\
\text { Garantizar el menor impacto en los } \\
\text { recursos que se requieren en cada } \\
\text { proyecto. }\end{array}$ & $\begin{array}{l}\text { - Reducir la accidentalidad, para todos. } \\
\text { Cumplir con la normativa. } \\
\text { - Mejorar o mantener las buenas } \\
\text { condiciones de seguridad y salud vs. } \\
\text { la labor. } \\
\text { - Fortalecer la seguridad y la salud } \\
\text { en la organización en cuanto a } \\
\text { participación. }\end{array}$ \\
\hline
\end{tabular}

Fuente: elaboración propia. 
es importante; eso es dar una calidad de vida a la gente; [...] yo le digo a mi equipo de trabajo: «realmente no sabemos cuántas vidas hemos salvado»". También la C2 reconoce la importancia en la mitigación del riesgo legal, un tema que aqueja a todos: "Sí, es esencial, precisamente por todo el tema que hemos hablado de mitigación del riesgo legal”. Sin embargo, la C3 da una opinión que va más allá, pues a pesar de estar de acuerdo con la gran importancia del SG-SST reconoce dos factores con los que no está de acuerdo:

1. Hay cosas que uno no comparte, como, por ejemplo, la forma como hacen las auditorías.

2. Yo hoy en día acepto el sistema de gestión, pero todos estos controles nos los están trasladando a nosotros, que es lo que dice el sistema: "usted tiene que velar por que el contratista cumpla".

f. Las consecuencias de no contar con un SG-SST fueron percibidas por las tres constructoras con los factores que se muestran en la figura 1 .

Figura 1. Factores que se dan por falta de un SG-SST

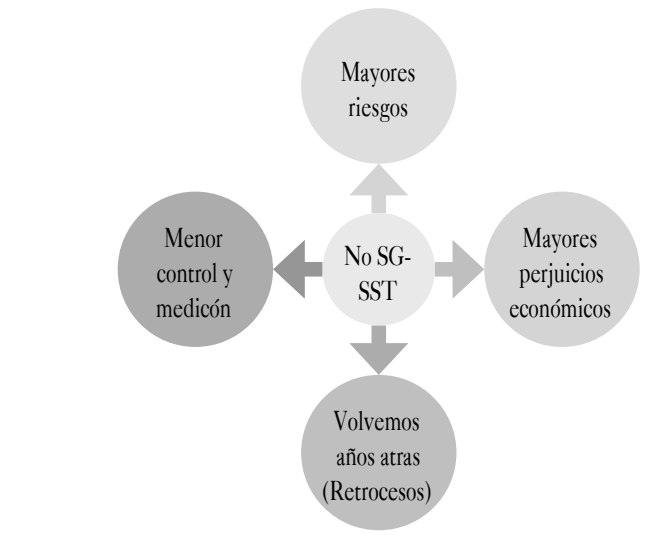

Fuente: elaboración propia.

\section{Enfoque de gerencia de proyectos}

Dentro de las preguntas enfocadas a la gerencia de proyectos, se busca conocer si las organizaciones reconocen el desarrollo del SG-SST como un proceso aliado a los componentes y fases de un proyecto, con el fin de lograr el éxito en el desarrollo de estos. Para este enfoque se desarrollaron tres preguntas.

a. La realización de un SG-SST debe generar 0 aportar factores de éxito a la compañía, permitiéndole cumplir sus objetivos y metas a corto, mediano o largo plazo. Las constructoras exponen sus factores de éxito del SG-SST en los proyectos así:

La C1 afirma: "Planeación, respaldo gerencial, asignación de recursos, anticipación a la evaluación del riesgo, el factor reputacional y legal"; la C2 manifiesta la importancia de tener metodologías como un factor de éxito clave dentro de las organizaciones, en especial en el desarrollo de un SG-SST, "pero definir metodologías, y claramente la forma de controlarlas, es un factor éxito"; por último, la C3 destaca una noción que ha venido desarrollando, las lecciones aprendidas: "las mismas lecciones aprendidas también son un tema de éxito".

b. En la planeación de cualquier proyecto, un factor fundamental es la identificación de factores internos y externos que puedan generar un impacto positivo o negativo en su desarrollo; en la tabla 4, se resumen estos factores. 
Tabla 4. Factores internos y externos para la implementación de un SG-SST

\begin{tabular}{|l|l|l|l|}
\hline Factores & \multicolumn{1}{|c|}{ C1 } & \multicolumn{1}{|c|}{ C2 } & \multicolumn{1}{c|}{ C3 } \\
\hline Internos & . Compromiso gerencial & $\begin{array}{l}\text { - Satisfacción del cliente (calidad) } \\
\text { Protección a la vida }\end{array}$ & $\begin{array}{l}\text { - Necesidades en el desarrollo de las } \\
\text { actividades } \\
\text { Experiencias vividas }\end{array}$ \\
\hline Externos & $\begin{array}{l}\text { Estudio del grado de implementación del } \\
\text { SG-SST por parte de otras constructoras } \\
\text { Legislación }\end{array}$ & $\begin{array}{l}\text { - Legislación } \\
\text { Protección al medio ambiente }\end{array}$ & - Legislación \\
\hline
\end{tabular}

Fuente: elaboración propia.

c. En la gerencia de proyectos, la comunicación y la divulgación toman un grado de importancia debido a que, a través de estos procesos, se incluyen otros que permiten asegurar la información, distribuirla de forma correcta y almacenarla para su permanencia como documentos de soporte, aprendizaje, conocimiento o experiencias. En este sentido, las constructoras entrevistadas manifestaron esta importancia así:

C1: "Tenemos una plataforma que se llama ARCU, arquitectura del conocimiento, donde se tienen cursos virtuales, charlas, etc.".

C2: "En la compañía tenemos un sistema de gestión documental".

C3: "Nosotros tenemos una tecnología que se llama Intranet, y es como una página, llamémoslo así, donde se publica todo lo de la organización; entonces, yo lo que hago es socializar allí temas del SG".

\section{Enfoque organizacional}

El SG-SST debe estar inmerso en todos los aspectos organizacionales de la constructora, pues busca asegurar y proteger un recurso de gran valor, el ser humano, la vida misma. Es así, como se enfocaron en el diagnóstico dos preguntas que apuntan al bienestar laboral y a la asignación de responsabilidades dentro del desarrollo de la dirección estratégica de la organización.

a. En cuanto al bienestar laboral, se contextualizó a los directivos con el concepto: Bienestar Laboral en los empleados comprende los beneficios que recibe el trabajador por parte de la empresa y otras entidades sin que constituyan el salario (Aguilar, Cruz, \& Jiménez, 2007. Con esta claridad, todas las constructoras manifestaron contar con programas de bienestar para sus empleados directos, aclarando también que el alcance de estos programas no llegaba a los contratistas dentro de los proyectos. Algunos ejemplos de estos programas fueron citados así:

C1: "Tenemos un programa de bienestar que se llama VITAL, tenemos la sala de lactancia, un programa de riesgo biomecánico, actividades como el día de la familia”.

C2: "A nivel de bienestar se trabaja, por ejemplo, en un torneo de bolos, fiesta de integración anual, se trabaja a nivel de toda la compañía, hay un manual de beneficios que integra todo el tema”.

C3: "[A nuestros empleado directos les ofrecemos] un programa de bienestar, donde lo que se [trata] es de promover que tengamos tiempo para la familia, que tengamos otro tipo de beneficios; por ejemplo, en el tema de salud, la organización [vela por] que los trabajadores tengan programas complementarios de salud, convenios que hay con entidades, como, por ejemplo, con gimnasios, con el cine, a través de puntos, entre otros".

b. Adicionalmente, se indagó acerca de la asignación de roles y responsabilidades del SG-SST en la ejecución de los proyectos, tomando en cuenta que la ley obliga a que los empleadores asignen y comuniquen las responsabilidades dentro del SG-SST, a todos los 
niveles, incluida la alta dirección. Es así, como las constructoras compartieron cómo realizaban esta asignación:

C1: "Lo que hicimos fue coger por niveles jerárquicos, definirles unas funciones y responsabilidades frente al SG-SST"

C2: "Las responsabilidades y los roles están atados a un tema; son socializados desde el proceso de inducción de los colaboradores. Es el tema principal, para que la compañía transmita qué es lo que se está esperando del desempeño de ese colaborador y que así queda atado precisamente a las responsabilidades. Otra forma como se integró hace parte del reglamento interno del trabajo: el tema de esas responsabilidades que vienen desde el Decreto 1072, y están atadas también a un perfil de cargo".

C3: "Hay unas actividades que son compartidas; obviamente, el mismo análisis del riesgo de las actividades involucran que estén los residentes, que también estén los maestros, pero todo eso lo lidera el residente de SST del proyecto".

\section{Enfoque de metodologías gerenciales}

Por último, se orientó una pregunta enfocada a la consideración del problema central de la investigación: la falta de incursionar en metodologías que les permitan a las constructoras aplicar e implementar con gran eficacia el SG-SST en los proyectos que desarrollan. Con este interrogante se buscaba conocer si recurrían a metodologías para implementar en los proyectos el SGSST, pues el hecho de ejecutarlo en cada proyecto no es una tarea fácil, pero con el apoyo del desarrollo de una metodología se considera más realizable y aterrizado el hecho de lograrlo.

a. Desarrollar metodologías permite que las compañías, organizaciones y, en este caso, las constructoras puedan alcanzar con mayor eficiencia los objetivos y las metas que se quieran conseguir; esta la razón es por la cual consideramos importante indagar sobre la utilización de dichas metodologías. Encontramos dentro de la muestra una de las tres que poseen medianamente algo que se puede llamar metodología, tal como lo exponen ellas mismas:

C1: "No, pues obviamente él no; para eso estamos nosotros, nosotros como área de ssT somos los que tenemos las herramientas para medir la gestión de seguridad y salud en el trabajo de la operación y de los contratistas". [Fin de cita]

La C2 por su parte es la única que manifestó tener algo parecido a una metodología, pero lo llama un plan de calidad y lo define así:

Sí hay un plan de calidad que también está entregado al tema de seguridad y salud, y al sistema ambiental; esa es la herramienta parte desde que un proyecto inicia, desde la etapa de la planificación.

La C3 finalmente indica no poseer una metodología y reconoce la necesidad de poseerla:

Pero como tal una metodología, que exista un mapeo donde le diga al gerente o donde le diga al coordinador de obra "yo tengo que hacer esto, esto y esto", no, estructuralmente, no. Sería bueno tenerlo. [Fin de cita]

\section{CONCLUSIONES}

El diagnóstico realizado a través de los cuatro enfoques permite confirmar las tres posiciones que sustentan el objetivo de esta investigación: el SG-SST necesita más investigación multidisciplinaria; es necesario continuar con el desarrollo de instrumentos que permitan medir la gestión en las organizaciones y la existencia de intervalos vacíos a nivel teórico y conceptual con relación al SG-SST.

En cuanto al enfoque del SG-SST, se puede percibir que, para las constructoras, el ámbito legal es uno de los aspectos que influye más al momento de implementarlo, pues lo exponen como factor fundamental para desarrollar los mismos. Adicionalmente, el aporte de la C3, con respecto a los controles que se tienen para con los contratistas en los proyectos de obra civil y el traslado de 
la responsabilidad a las constructoras, es un aspecto que el gobierno debe revaluar para que no sea interpretado como un traslado de responsabilidad, sino como una corresponsabilidad, en donde una parte cuida a otra y viceversa (constructora-contratista).

La NTC-ISO 45001:2018 trae consigo cambios muy importantes, que brindan lineamientos metodológicos para la implementación del SG-SST; por eso, aunque no sea de interés de la constructora certificar su sistema de gestión, guiarse por estos aspectos, conocerlos y valorarlos fortalecen y enriquecen los procesos encaminados a la seguridad y salud del recurso humano.

En cuanto al enfoque de gerencia de proyectos, un factor de éxito que se debe destacar es el de las lecciones aprendidas, que generan cada día más valor, pues el conocimiento adquirido a través de las experiencias se debe recopilar, compilar, conservar y divulgar, con el fin de mantener los casos de éxito, como lineamientos a seguir, y los fracasos o errores para no volver a repetirlos.

Seguidamente, el enfoque organizacional permite a las organizaciones generar estrategias y planes de bienestar para asegurar aún más la calidad de vida de las personas, ofreciendo reconocimientos a su labor a través de elementos adicionales diferentes al salario. En este sentido, las constructoras entrevistadas tienen claro que el incentivo laboral a través de planes de bienestar es un soporte fundamental para el SG-SST.

Por último, las constructoras confirman que contar con una metodología que les permita implementar con mayor facilidad, agilidad y eficacia el SG-SST en los proyectos que desarrollan es importante y necesario para obtener mayor cobertura y recepción por parte de todos los stakeholders, logrando con ellos el mejoramiento continuo del sistema.

\section{REFERENCIAS}

Acevedo, H., Vásques, A., \& Ramírez, D. (2012). Sostenibilidad: Actualidad y necesidad en el sector de la construcción en Colombia. Gestión y Ambiente, 15(1), 105-118. Recuperado de https://revistas.unal.edu. co/index.php/gestion/article/view/30825/30933

Aguilar, V., Cruz, D., \& Jiménez, D. (2007). Bienestar social laboral desde la perspectiva de calidad de vida a partir de la producción escrita y la percepción de docentes especialistas. 1995-2005 en Bogotá, D.C. Estado del arte. Revista Tendencia \& Retos. 12, 247249

Alarcón, L., Acuña, D., Diethelm, S., \& Pellicer, E. (2016). Strategies for improving safety performance in construction firms. Accident Analysis \& Prevention, 94, 107-118. DOI: https://doi.org/10.1016/j. aap.2016.05.021

Así quedó el balance de accidentes y enfermedades laborales en 2017. (15 de febrero de 2018). Revista Dinero. Recuperado de https://www.dinero.com/ edicion-impresa/pais/articulo/accidentes-y-enfermedades-laborales-en-2017/255313

Cagno, E., Micheli, G., Masi, D., \& Jacinto, C. (2012). Economic evaluation of OSH and its way to SMEs: A constructive review. Safety Science, 53, 134-152. DoI: https://doi.org/10.1016/j.ssci.2012.08.016

Clavijo, S. (2018). Desempeño sector construcción y sus encadenamientos sectoriales. La Repúblicas. Recuperado de https://www.larepublica.co/analisis/ sergio-clavijo-500041/desempeno-sector-construccion-y-sus-encadenamientos-sectoriales-2752867

Erazo, G., \& Rodríguez-Rojas, Y. (2016). Diseño y validación de contenido de un instrumento para medir 
la gestión de la seguridad y salud en el trabajo para organizaciones colombianas. Signos - Investigación en sistemas de gestion, 8(2), 65-80. DoI: https://doi. org/10.15332/s2145-1389.2016.0002.03

Hernández-Sampieri, R., \& Mendoza, C. (2018). Metodología de la investigación (8ta ed.). México D.F, México: Mc Graw Hill.

Ministerio de Trabajo. (2018). Decreto número 1072 de 2015, por el cual se expide el Decreto Único. Bogotá: Ministerio de Trabajo.

Rodríguez, Y., Pedraza, X., \& Martínez, J. (2017). Evaluación de la madurez de la gestión de la seguridad y salud en el trabajo: revisión de literatura. SIGNOS Investigación en Sistemas de Gestión, 9(1), 113-127. DoI: https://doi.org/10.15332/s2145-1389.2017.0001.08

SafetYA. (2019). Accidentes de trabajo en Colombia en cifras. Recuperado de https://safetya.co/accidentes-detrabajo-en-colombia-en-cifras-2018/
Top 5 de las constructoras más importantes de Colombia 2017. (2020). Infoconstrucción LATAM. Recuperado de https://infoconstruccionlatam.com/top-5-de-lasconstructoras-mas-importantes-de-colombia-2017/

Top 5: derrumbes de estructuras por errores en la construcción. (16 de enero de 2018). Publimetro. Recuperado de https://www.publimetro.co/co/colombia/2018/01/16/top-5-derrumbes-estructuraserrores-la-construccion.html

Vicepresidencia de Colombia. (2015). La construcción sigue siendo el motor de la economía Colombiana: Vargas Lleras. Recuperado de http://www.vicepresidencia. gov.co/prensa/2015/Paginas/La-construccion-sigue-siendo-el-motor-de-la-economia-colombiana-150910.aspx

Zhou, Z., Goh, M., \& Li, Q. (2014). Overview and analysis of safety management studies in the construction industry. Safety Science, 72, 337-350. Dor: https://doi. org/10.1016/j.ssci.2014.10.006 